\title{
Single lung ventilation in patients undergoing lobectomy
}

\author{
Abdurahim Aloud, Gilbert Berdine, Kenneth Nugent \\ Department of Internal Medicine at Texas Tech University Health Sciences Center, Lubbock, TX, USA \\ Correspondence to: Kenneth Nugent. Department of Internal Medicine at Texas Tech University Health Sciences Center, Kenneth Nugent, 3601 4th \\ Street, Lubbock, TX 79430, USA. Email: kenneth.nugent@ttuhsc.edu. \\ Comment on: Gao S, Zhang Z, Brunelli A, et al. The Society for Translational Medicine: clinical practice guidelines for mechanical ventilation \\ management for patients undergoing lobectomy. J Thorac Dis 2017;9:3246-54.
}

Submitted Oct 29, 2018. Accepted for publication Oct 31, 2018.

doi: $10.21037 /$ jtd.2018.11.05

View this article at: http://dx.doi.org/10.21037/jtd.2018.11.05

The outcome of patients undergoing lobectomy will depend, in part, on preexistent lung disease and lung function. These patients often have chronic obstructive pulmonary disease (COPD) and reduced lung function. In addition, they will necessarily lose lung volume during the surgical procedure. Consequently, complications will depend on preoperative lung function, intraoperative management, and postoperative care. The first step in their management requires careful preoperative assessment and treatment of any reversible lung disease, such as infection and bronchospasm. The second step requires selection of the planned operative procedure. This likely depends on the anatomic distribution of the disease, the surgeon's skills, and the surgeon's experience. The third step involves intraoperative management with single lung ventilation resulting in collapse of the ipsilateral lung and ventilation of the contralateral lung to maintain gas exchange during the procedure. This ventilatory strategy necessarily results in atelectasis, produces abnormal gas exchange with possible hypoxemia and/or hypercapnia, and potentially causes overinflation of the contralateral lung $(1,2)$. Therefore, during a single procedure, the patient is at risk for atelectrauma, volutrauma, and biotrauma (2). We will discuss these considerations in patients undergoing lobectomy and comment on the guideline "The Society for Translational Medicine: clinical practice guidelines for mechanical ventilation management for patients undergoing lobectomy" published in the Fournal of Thoracic Disease (3).

\section{Complications}

Video-assisted thoracoscopic surgery (VATS) has potential benefits over thoracotomy for lung resection; these include faster patient recovery times, fewer complications, and shorter hospital stays without compromising the oncologic aspects of the operation (4). Several studies have shown that long-term survival and locoregional recurrence in patients operated on by VATS are comparable to those in patients who underwent lobectomy by thoracotomy. However, VATS lobectomy for the treatment of resectable lung cancer does have complications. Patients undergoing VATS were compared to patients undergoing thoracotomy in the American College of Surgeons Oncology Group Z0030 randomized trial. Atelectasis requiring therapeutic bronchoscopy occurred less frequently in the VATS group, and chest tube drainage lasting more than 7 days occurred less frequently in the VATS patients (4). These results may reflect decreased pain and better mobilization of secretions secondary to a stronger cough because of improved postoperative chest wall function in the VATS lobectomy group. The frequency of other complications was similar in the two cohorts in this study (4).

The complication rates following VATS lobectomy for lung cancer range between $6 \%$ and $34.2 \%$ and the mortality rate ranges between $0.6 \%$ and $1.3 \%(5,6)$. The reported incidence of these complications varies widely in different studies; Wang et al. reported prolonged air leak $(7.6 \%)$, pneumonia (5\%), pleural effusion $(3.1 \%)$, pulmonary atelectasis $(6.4 \%)$, acute respiratory distress syndrome $(0.7 \%)$, and ventilator dependence $(1.0 \%)$ in a study involving 828 patients (7). Prolonged air leak and atelectasis were the most common complications. These complications, acute respiratory distress syndrome, and respiratory failure requiring mechanical ventilation for 
longer than 24 hours could be related to the ventilator management during anesthesia.

The following studies provide more detailed information about complications during VATS procedures. Sandri et al. analyzed major morbidity in 227 patients undergoing VATS for lung resection (5). They compared complications using the European Society of Thoracic Surgeons' (ESTS) classification with complications based on the Thoracic Morbidity and Mortality system (TM\&M) for classification. The ESTS protocol defines two classes of complications: major and minor. Major complications are defined as adverse events that require surgical, radiologic, endoscopic treatment, multi-therapy with or without general anesthesia, or intensive care unit treatment for single or multiple organ dysfunction. Thirty-four patients in this study had complications. Based on the ESTS definitions, 20 patients had major complications, including atrial fibrillation [10], adult respiratory distress syndrome [1], pulmonary embolus [2], prolonged mechanical ventilation [1], pneumonia [3], and atelectasis requiring bronchoscopy [2]. Based on the TM\&M system, 24 patients had major complications, including 3 deaths. In this study 18 patients $(7.9 \%)$ required conversion from a VATS procedure to an open thoracotomy.

Lai et al. recorded the postoperative pulmonary complications in 725 patients undergoing lobectomy for a lung cancer (8). Postoperative complications within 30 days occurred in 144 patients. These included pneumonia (87, $12.0 \%$ of total patients), atelectasis (41, 5.7\%), pulmonary air leak $(37,5.1 \%)$, mechanical ventilation for greater than 48 hours (24, 3.3\%), empyema (14, 1.9\%), and respiratory/ cardiac failure or acute respiratory distress syndrome $(16,2.2 \%)$. Patients with a preoperative peak expiratory flow rate less than $300 \mathrm{~L} / \mathrm{min}$ had an increased rate of complications. Pompili et al. analyzed 5,069 patients undergoing lobectomy to determine the factors associated with prolonged air leak defined as longer than 5 days (9). Prolonged air leak occurred in 504 patients (9.9\%). Factors associated with prolonged air leak included male gender, FEV1 less than $80 \%$ of predicted, and a body mass index less than $18.5 \mathrm{~kg} / \mathrm{m}^{2}$. These authors suggested that this information might allow surgeons to make changes in intraoperative procedures to reduce the rate of air leak in high risk patients.

\section{Surgical procedure}

The VATS procedure necessarily causes chest wall and lung trauma that can lead to the release of inflammatory mediators. This results in biotrauma and can have both local pulmonary effects and systemic effects $(1,2,10)$. Longer surgical procedures increase the frequency of postoperative complications. In addition, surgical resections cause air leaks that occur in approximately $10 \%$ of patients. These have been associated with reduced pulmonary function, lower body mass indices (which probably reflects malnutrition), and pleural adhesions (9). The surgical procedure necessarily leaves patients with chest tubes, which increases the risk for pleural space infections. Both air leaks and pleural space infections prolong hospitalization and increase medical costs.

\section{Single lung ventilation}

Ventilation of the contralateral lung during lobectomies provides the minute ventilation necessary for oxygenation and $\mathrm{CO}_{2}$ excretion $(1,2)$. However, this lung is at risk for volume trauma if the tidal volume overexpands more normal regions of the lung. In addition, this lung is in a dependent position and often develops atelectasis. Regions in which the normal lung is adjacent to the atelectatic lung may have more mechanical stress during the respiratory cycle with cyclical opening and closure in the atelectatic lung and overinflation in the normal lung. This can result in epithelial injury, the release of inflammatory mediators, and the development of acute respiratory failure. In addition, this lung is at risk for pneumothorax, especially if the patient has underlying lung disease, such as COPD. Consequently, the anesthesiologist needs to consider the best ventilatory parameters for adequate oxygenation with the lowest possible airway and alveolar pressures. Volatile anesthetics may reduce the release of cytokines and other inflammatory mediators during anesthesia (2).

\section{Ipsilateral atelectasis}

Atelectasis is common following pulmonary resection and has a wide range in incidence from $1-20 \%$, probably because "clinically significant atelectasis" has no standard definition. Factors involved in the pathogenesis of post-lobectomy atelectasis include abnormal mucociliary function, chronic tobacco use, COPD, increasing age, longer operating time, and inadequate postoperative pain management (11). A high American Society of Anesthesiologists (ASA) score is an important risk factor for postoperative complications, independent of the operative procedure, and high ASA 
scores have been associated with postoperative pneumonia and prolonged postoperative intubation.

Korst et al. and Uzieblo et al. reported that patients undergoing right upper lobe (RUL) resection are at a higher risk for post-lobectomy atelectasis $(12,13)$. The reason for this predisposition is unclear but probably relates to anatomic factors. After RUL resection, the lower lobe expands to fill the right hemithorax which may result in anatomic distortion of the bronchi by kinking. Postlobectomy atelectasis in the collapsed lung occurs most frequently on post-operative day 2 and 3. Contralateral atelectasis occurs earlier than its ipsilateral atelectasis, suggesting different mechanisms. In contralateral atelectasis, the pooling of secretions in the dependent lung during surgery is a possible factor. However, Jaworski et al. concluded that the intraoperative collection of secretions was not the major cause of atelectasis, since immediate postoperative bronchoscopy did not prevent its occurrence (14).

The use of bronchoscopy to clear retained secretions has become a standard procedure for patients with atelectasis, and this is effective in $70 \%$ to $89 \%$ of patients. Patients with lobar atelectasis respond better than those with subsegmental atelectasis, probably because they have large central plugs that can be removed by bronchoscopy. Patients undergoing lobectomy need postoperative physiotherapy. The most common techniques used by physiotherapists include positioning, mobilization, manual hyperinflation, and breathing exercises. The value of adding incentive spirometry to physiotherapy is controversial. Adequate pain control is an important factor in postoperative management.

The collapsed lung on the surgical side develops significant atelectasis and then reduced blood flow resulting in ischemia (15-17). This place this lung in jeopardy for developing ischemia-reperfusion syndrome with lung injury. In addition, the alveolar oxygen level in this lung drops to very low levels (alveolar hypoxia). When the lung is reexpanded, there is rapid re-oxygenation that can also produce reactive oxygen species. These metabolic events can cause injury to the atelectatic lung and to the contralateral lung and can cause systemic injury. In some patients this leads to diffuse pulmonary infiltrates, acute respiratory failure, and the acute respiratory distress syndrome.

\section{Acute respiratory failure}

Multiple factors, including preexistent lung disease, surgical resection, ventilator-associated injury, and atelectasis, can cause acute respiratory failure requiring prolonged mechanical ventilation following lobectomy. Some of these patients develop diffuse infiltrates consistent with the acute respiratory distress syndrome. The pathogenesis involves the release of inflammatory mediators, damage to alveolar structures during cyclical opening and closing or during overinflation, reperfusion injury following recovery of blood flow to the atelectatic lung, and possibly oxygen radicals secondary to high $\mathrm{O}_{2}$ concentrations causing direct injury. The mortality rate is significantly increased in these patients.

\section{Guidelines}

The Society for Translational Medicine guidelines on mechanical ventilation in these patients included the following recommendations (3). Permissive or therapeutic hypercapnia to maintain a $\mathrm{PaCO}_{2}$ of $50-70 \mathrm{mmHg}$ may be beneficial in these patients. Ventilation with a tidal volume of $6-8 \mathrm{~mL} / \mathrm{kg}$ and a PEEP level of $5 \mathrm{cmH}_{2} \mathrm{O}$ is reasonable. Alveolar recruitment and the use of a low $\mathrm{FiO}_{2}$ may prevent absorption atelectasis in the ventilated lung. The use of adjuvant medications, such as preoperative nebulized budesonide, may improve lung mechanics and reduce inflammation in the ventilated lung (18). All these guidelines were rated as Class IIa or Class IIb at Levels A-C, indicating that the benefit exceeded the risk but additional studies are needed.

\section{Recommendations}

In summary, the complications associated with single lung ventilation for lobectomy include complications associated with the surgical procedure, complications associated with single lung ventilation of the dependent contralateral lung, and complications associated with non-ventilation of the ipsilateral collapsed lung. Thoracic surgeons and anesthesiologists have two important concerns during these surgical procedures. These include adequate oxygenation during the surgical procedure and the potential for the development of acute lung injury in either the ipsilateral collapsed lung or the contralateral ventilated lung or both lungs. The low pressure, low tidal volume approach to the management of patients with acute lung injury should be used by anesthesiologists in the management of patients requiring one lung ventilation $(10,19)$. The initial ventilator parameters should include a low tidal volume $(6 \mathrm{~mL} / \mathrm{kg})$, a low plateau pressure $\left(30 \mathrm{cmH}_{2} \mathrm{O}\right)$, and the lowest possible 
$\mathrm{FiO}_{2}$ to maintain $\mathrm{O}_{2}$ saturations in the $92-95 \%$ range. Even though a tidal volume of $6 \mathrm{~mL} / \mathrm{kg}$ seems relatively low, it should be remembered that this is a tidal volume for a single lung. The ventilated lung is in a dependent position and develops some atelectasis. Therefore, recruitment maneuvers and PEEP should be used to limit this development. The collapsed lung on the surgical side is, of course, atelectatic, and continuous positive airway pressure (CPAP) applied to this lung partially limits this development. The introduction of a catheter into the collapsed nonventilated lung can provide supplemental oxygen, if needed. The development of some hypercapnia during the management of these patients does not represent a significant risk except in certain clinical circumstances, such as patients with intracranial hypertension, patients with pulmonary hypertension, and patients with unstable cardiovascular status. The use of volatile anesthetics may reduce the inflammatory response associated with these procedures (2). Finally, the postoperative use of noninvasive positive pressure ventilation may help stabilize patients until they recover from the surgical procedure (20).

\section{Acknowledgements}

None.

\section{Footnote}

Conflicts of Interest: The authors have no conflicts of interest to declare.

\section{References}

1. Meleiro H, Correia I, Charco Mora P. New evidence in one-lung ventilation. Rev Esp Anestesiol Reanim 2018;65:149-53

2. Şentürk M, Slinger P, Cohen E. Intraoperative mechanical ventilation strategies for one-lung ventilation. Best Pract Res Clin Anaesthesiol 2015;29:357-69.

3. Gao S, Zhang Z, Brunelli A, et al. The Society for Translational Medicine: clinical practice guidelines for mechanical ventilation management for patients undergoing lobectomy. J Thorac Dis 2017;9:3246-54.

4. Scott WJ, Allen MS, Darling G, et al. Video-assisted thoracic surgery versus open lobectomy for lung cancer: a secondary analysis of data from the American College of Surgeons Oncology Group Z0030 randomized clinical trial. J Thorac Cardiovasc Surg 2010;139:976-81; discussion 981-3.

5. Sandri A, Papagiannopoulos K, Milton R, et al. Major morbidity after video-assisted thoracic surgery lung resections: a comparison between the European Society of Thoracic Surgeons definition and the Thoracic Morbidity and Mortality system. J Thorac Dis 2015;7:1174-80.

6. Ziarnik E, Grogan EL. Postlobectomy Early Complications. Thorac Surg Clin 2015;25:355-64.

7. Wang S, Li X, Li Y, et al. The long-term impact of postoperative pulmonary complications after video-assisted thoracic surgery lobectomy for lung cancer. J Thorac Dis 2017;9:5143-52.

8. Lai Y, Wang X, Li P, et al. Preoperative peak expiratory flow (PEF) for predicting postoperative pulmonary complications after lung cancer lobectomy: a prospective study with 725 cases. J Thorac Dis 2018;10:4293-301.

9. Pompili C, Falcoz PE, Salati M, et al. A risk score to predict the incidence of prolonged air leak after videoassisted thoracoscopic lobectomy: An analysis from the European Society of Thoracic Surgeons database. J Thorac Cardiovasc Surg 2017;153:957-65.

10. Kozian A, Schilling T, Schütze H, et al. Ventilatory protective strategies during thoracic surgery: effects of alveolar recruitment maneuver and low-tidal volume ventilation on lung density distribution. Anesthesiology 2011;114:1025-35.

11. Stolz AJ, Schutzner J, Lischke R, et al. Predictors of atelectasis after pulmonary lobectomy. Surg Today 2008;38:987-92.

12. Korst RJ, Humphrey CB. Complete lobar collapse following pulmonary lobectomy. Its incidence, predisposing factors, and clinical ramifications. Chest 1997;111:1285-9.

13. Uzieblo M, Welsh R, Pursel SE, et al. Incidence and significance of lobar atelectasis in thoracic surgical patients. Am Surg 2000;66:476-80.

14. Jaworski A, Goldberg SK, Walkenstein MD, et al. Utility of immediate postlobectomy fiberoptic bronchoscopy in preventing atelectasis. Chest 1988;94:38-43.

15. Della Rocca G, Coccia C. Acute lung injury in thoracic surgery. Curr Opin Anaesthesiol 2013;26:40-6.

16. Tusman G, Böhm SH, Warner DO, et al. Atelectasis and perioperative pulmonary complications in high-risk patients. Curr Opin Anaesthesiol 2012;25:1-10.

17. Heerdt PM, Stowe DF. Single-lung ventilation and oxidative stress: a different perspective on a common practice. Curr Opin Anaesthesiol 2017;30:42-9.

18. Ju NY, Gao H, Huang W, et al. Therapeutic effect 
of inhaled budesonide (Pulmicort@ Turbuhaler) on the inflammatory response to one-lung ventilation. Anaesthesia 2014;69:14-23.

19. Brassard CL, Lohser J, Donati F, et al. Step-bystep clinical management of one-lung ventilation: continuing professional development. Can J Anaesth
2014;61:1103-21.

20. Okada S, Ito K, Shimada J, et al. Clinical application of postoperative non-invasive positive pressure ventilation after lung cancer surgery. Gen Thorac Cardiovasc Surg 2018;66:565-72.

Cite this article as: Aloud A, Berdine G, Nugent K. Single lung ventilation in patients undergoing lobectomy. J Thorac Dis 2018;10(12):6383-6387. doi: 10.21037/jtd.2018.11.05 